\title{
Enhancement of supersymmetry near a 5D black hole horizon
}

\author{
Ali Chamseddine* \\ Theoretishe Physik, ETH-Hönggenberg, CH-8093 Zúrich, Switzerland \\ Sergio Ferrara ${ }^{\dagger}$ \\ Theory Division, CERN, 1211 Geneva 23, Switzerland \\ Gary W. Gibbons \\ DAMTP, Cambridge University, Silver Street, Cambridge CB3 9EW, United Kingdom \\ Renata Kallosh ${ }^{\S}$ \\ Physics Department, Stanford University, Stanford, California 94305-4060
}

(Received 29 October 1996)

\begin{abstract}
Geometric Killing spinors which exist on $\mathrm{AdS}_{p+2} \times S^{d-p-2}$ sometimes may be identified with supersymmetric Killing spinors. This explains the enhancement of unbroken supersymmetry near the $p$-brane horizon in $d$ dimensions. The corresponding $p$-brane interpolates between two maximally supersymmetric vacua, at infinity and at the horizon. A new case is studied here: $p=0, d=5$. The details of the supersymmetric version of the very special geometry are presented. We find the area-entropy formula of the supersymmetric 5D black holes via the volume of $S^{3}$ which depends on charges and the intersection matrix. [S0556-2821(97)00406-2]
\end{abstract}

PACS number(s): 04.70.Dy, 04.50.+h, 11.30.Pb

\section{INTRODUCTION}

The enhancement of supersymmetry near the $p$-brane horizon is an interesting phenomenon. The corresponding $p$-branes interpolate between two maximally supersymmetric vacua: $\mathcal{M}^{d}$ at infinity and $\operatorname{AdS}_{p+2} \times S^{d-p-2}$ near the horizon [1-3]. The generic reason for the enhancement of supersymmetry is the following. Any anti-de Sitter space as well as any sphere admit Killing spinors which we will call geometric Killing spinors. The relation of this geometric Killing spinor, which has a dimension of a Dirac spinor, to the Killing spinor of unbroken supersymmetry requires further investigation. In theories with $N=2$ supersymmetry the unbroken supersymmetry of the $p$-brane has dimension of one-half of the Dirac spinor. If near the horizon the dimension of the supersymmetric Killing spinor becomes that of the Dirac spinor, we have an enhancement of supersymmetry. In some cases it has already been established that the Killing spinor defined by the zero mode of the gravitino transformation at the near horizon geometry of the $p$-brane solutions coincides with the geometric spinors. These cases include $d=4, p=0$ with $\operatorname{AdS}_{2} \times S^{2}$ [1,2], $d=10, p=3$ with $\mathrm{AdS}_{5} \times S^{5}, d=11, p=2$ with $\mathrm{AdS}_{3} \times S^{7}$, and $d=11, p=5$ with $\mathrm{AdS}_{7} \times S^{3}$ [3]. The near horizon geometry of these $p$-branes is known to be maximally supersymmetric. In $d=4$ the integrability condition for the Bertotti-Robinson geometry near the black hole horizon $\operatorname{AdS}_{2} \times S^{2}$ was proved in $[4,2]$ using the fact that this geometry is conformally flat and that the graviphoton field strength is covariantly constant.

\footnotetext{
*Electronic address: chams@itp.phys.ethz.ch

${ }^{\dagger}$ Electronic address: ferraras@cernvm.cern.ch

‡Electronic address: G.W.Gibbons@damtp.cam.ac.uk

${ }^{\S}$ Electronic address: kallosh@renata.stanford.edu
}

Remarkably, the supersymmetry near the fivedimensional (5D) black hole horizon has not yet been studied. Moreover, the unbroken supersymmetry of the 5D black holes was established in [5] only for black holes of pure $N=2, d=5$ supergravity without vector multiplets. In particular, the Strominger-Vafa 5D black holes [6] and the rotating generalization of them [7] have not yet been embedded into a particular 5D supersymmetric theory and the unbroken supersymmetry of either solution has not been checked directly. An analogous situation holds with the more general 5D static and rotating black holes found in [8]. There are various indications, however, that these solutions have unbroken supersymmetry.

The purpose of this paper is to find out whether the fivedimensional black holes near the horizon show the enhancement of the supersymmetry. Specifically, we will try to identify the supersymmetric Killing spinors admitted by black holes near the horizon with the geometric Killing spinors of $\mathrm{AdS}_{2} \times S^{3}$. We will also derive the area formula for generic solutions in $N=2$ theory interacting with an arbitrary number of vector multiplets as the volume of the $S^{3}$ and express it as the function of charges and the intersection matrix.

For the $d$-dimensional manifold which is a product space $\operatorname{AdS}_{p+2} \times S^{d-p-2}$ the geometric Killing spinors are given by the product of Killing spinors on $\operatorname{AdS}_{p+2}$ and $S^{d-p-2}$. On $\operatorname{AdS}_{p+2}$ and on $S^{d-p-2}$ the Killing spinor equations are

$$
\begin{gathered}
\hat{\nabla}_{a} \eta(x) \equiv\left(\nabla_{a}+c_{1} \tilde{\gamma}_{a}\right) \eta(x)=0, \quad a=0,1, \ldots, p+1, \\
\hat{\nabla}_{\alpha} \varsigma(y) \equiv\left(\nabla_{\alpha}+c_{2} \gamma_{\alpha}\right) \varsigma(y)=0, \quad \alpha=p+2, \ldots, d-1 .
\end{gathered}
$$

Here $\gamma_{\alpha}$ is the $\gamma$ matrix of the $(d-p-2)$-dimensional Euclidean space and the commutator $\left[\widetilde{\gamma}_{a}, \widetilde{\gamma}_{b}\right]$ equals 
$-\left[\gamma_{a}, \gamma_{b}\right]$ where $\gamma_{a}$ is the $\gamma$ matrix of the $(p+2)$-dimensional Minkowski space.

The integrability conditions for the geometric Killing spinors defined above are

$$
\left[\hat{\nabla}_{a}, \hat{\nabla}_{b}\right] \eta(x)=0 \Rightarrow R_{a b}{ }^{c d}=4\left(c_{1}\right)^{2}\left(\delta_{a}^{c} \delta_{b}{ }^{d}-\delta_{a}{ }^{d} \delta_{b}{ }^{c}\right)
$$

$$
\left[\hat{\nabla}_{\alpha}, \hat{\nabla}_{\beta}\right] \mathrm{s}(y)=0 \Rightarrow R_{\alpha \beta}^{\gamma \delta}=-4\left(c_{2}\right)^{2}\left(\delta_{\alpha}^{\gamma} \delta_{\beta}^{\delta}-\delta_{\alpha}{ }^{\delta} \delta_{\beta}^{\gamma}\right)
$$

and it is satisfied by the geometries of the anti-de Sitter space and sphere. The full Killing spinor is

$$
\epsilon(x, y)=\eta(x) \varsigma(y)
$$

and it forms a Dirac spinor in a given dimension $d$. On the other hand, the Killing spinor of unbroken supersymmetry of the $p$-brane solution near the horizon is defined by the corresponding supersymmetry transformation of the gravitino. If supersymmetric Killing spinor near the horizon can be identified with the geometric one of the maximally supersymmetric product space, we have an enhancement of supersymmetry:

$$
\delta \Psi_{M}=0 \Rightarrow \hat{\nabla}_{M} \epsilon(x, y)=0 \Rightarrow\left(\begin{array}{c}
\hat{\nabla}_{\mu} \eta(x)=0 \\
\hat{\nabla}_{\alpha} \varsigma(y)=0
\end{array}\right)
$$

To study this issue we will work out some details of $d=5, N=2$ supergravity coupled to $N=2$ vector multiplets in the framework of very special geometry, see Sec. II. This formulation of the $d=5$ theory is particularly adapted to $d=11$ supergravity compactified on Calabi-Yau manifold characterized by the intersection number $C_{A B C}$. In Sec. III we will identify the supersymmetric Killing spinors admitted by black holes near the horizon with the geometric Killing spinors of $\mathrm{AdS}_{2} \times S^{3}$. We will also perform a detailed derivation of the area formula of the five-dimensional black holes [9] in terms of the volume of $S^{3}$ which we evaluate in terms of the charges and intersection number matrix. In Sec. IV we will consider a special case of the $N=2$ theory interacting with one vector multiplet, which comes from the truncation of $N=4, d=5$ supergravity. This will allow us to embed some of the known black holes into supersymmetric theories and study the enhancement of supersymmetry near the horizon. We also study the $N=4$ theory interacting with arbitrary number $n$ of $N=4$ vector multiplets as a particular example of $N=2$ theory interacting with $n+1, N=2$ vector multiplets and intersection matrix $C_{0 i j}=\eta_{i j}$, where $\eta_{i j}$ is the Lorenzian metric on $(1, n)$. The duality invariant areaentropy formula for the $N=4$ theory is truncated to $N=2$ theory as it was done before in four-dimensional theory in [10]. In this way one describes the theory with very special geometry $\mathrm{O}(1,1) \times \mathrm{O}(1, n) / \mathrm{O}(n)$. In the discussion section we explain the relation between the enhancement of supersymmetries and finiteness of the area of the horizon and speculate about other cases as yet not known.

\section{SUPERSYMMETRIC VERY SPECIAL GEOMETRY IN $\boldsymbol{d}=\mathbf{5}$}

The action for $d=5, N=2$ supergravity coupled to $N=2$ vector multiplets has been constructed by Günaydin, Sierra, and Townsend [11]. The bosonic part of the action has been adapted to the very special geometry [12] and the compactification of 11D supergravity down to five dimensions on Calabi-Yau threefolds [13] with Hodge numbers $\left(h_{(1,1)}, h_{(2,1)}\right)$ and topological intersection form $C_{I J K}$. For our purpose, it will be extremely useful to adapt the full action and the supersymmetry transformation laws of [11] to that of very special geometry. In what follows we will give the detailed derivation of some formulae reported in [9].

The fields of the theory are $e_{\mu}{ }^{m}, A_{\mu}{ }^{I}, \phi^{i}, \psi_{\mu r}, \lambda_{r}{ }^{i}$, where $I=0,1, \ldots, h_{(1,1)}-1, i=1, \ldots, h_{(1,1)}-1$, and $r=1,2$. The index $r$ on the spinors is raised and lowered with the symplectic metric $\epsilon_{r s}$ and will be omitted.

The $N=2, d=5$ supersymmetric Lagrangian describing the coupling of vector multiplets to supergravity is determined by one function which is given by the intersection form on a CY threefold:

$$
\mathcal{V}=\frac{1}{6} C_{I J K} X^{I} X^{J} X^{K}
$$

The action is

$$
\begin{aligned}
e^{-1} \mathcal{L}= & -\frac{1}{2} R-\frac{1}{2} \bar{\psi}_{\mu} \Gamma^{\mu \nu \rho} D_{\nu} \psi_{\rho}-\frac{1}{4} G_{I J} F_{\mu \nu}{ }^{I} F^{\mu \nu J} \\
& -\frac{i}{2} \bar{\lambda}^{i}\left(g_{i j} \Gamma^{\mu} D_{\mu}+\Gamma^{\mu} \partial_{\mu} \phi^{k} \Gamma_{k j}^{l} g_{l i}\right) \lambda^{j} \\
& -\frac{1}{2} g_{i j} \partial_{\mu} \phi^{i} \partial^{\mu} \phi^{j}-\frac{i}{2} \bar{\lambda}_{i} \Gamma^{\mu} \Gamma^{\nu} \partial_{\nu} \phi^{i} \\
& +\frac{1}{4}\left(\frac{3}{4}\right)^{2 / 3} t_{I, i} \bar{\lambda}^{i} \Gamma^{\mu} \Gamma^{\lambda \rho} \psi_{\mu} F_{\lambda \rho}^{I}+\frac{i}{16 \cdot 6^{1 / 3}} \\
& \times\left(g_{i j} t_{I}-9 C_{J K L} t_{, i}^{J} t_{, j}^{K} t_{, k}^{L} t_{I}^{, k}\right)-\frac{3 i}{16 \cdot 6^{1 / 3} t_{I}} \\
& \times\left(\bar{\psi}_{\mu} \Gamma^{\mu \nu \rho \sigma} \psi_{\nu} F_{\rho \sigma}^{I}+2 \bar{\psi}^{\mu} \psi^{\nu} F_{\mu \nu}^{I}\right)+\frac{e^{-1}}{48} \epsilon^{\mu \nu \rho \sigma \lambda} \\
& \times C_{I J K} F_{\mu \nu}^{I} F_{\rho \sigma}^{J} A_{\lambda}^{K}+\cdots,
\end{aligned}
$$

where we use dots for four-fermionic terms. Here $t^{I}=t^{I}(\phi)$ are the special coordinates subject to the conditions

$$
t^{I} t_{I}=1, \quad C_{I J K} t^{I} t^{J} t^{K}=1,
$$

and $t_{I}$ are the "dual coordinates"

$$
t_{I}=C_{I J K} t^{J} t^{K} \equiv C_{I J} t^{J}, \quad t^{I}=C^{I J} t_{J} .
$$

We have introduced a notation

$$
C_{I J} \equiv C_{I J K} t^{K}, \quad C^{I J} C_{J K}=\delta_{K}^{I} .
$$

The metric is derived from the prepotential (7) through the relation $\left(\partial_{I} \equiv \partial / \partial X^{I}\right)$ 


$$
G_{I J}=-\left.\frac{1}{2} \partial_{I} \partial_{J}(\ln \mathcal{V})\right|_{\mathcal{V}=1}
$$

and $X^{I}$ is related to $t^{I}$ by

$$
X^{I}=\left.6^{1 / 3} t^{I}\right|_{\mathcal{V}=1} .
$$

Finally, the metric $g_{i j}$ is given by

$$
g_{i j}=G_{I J} X^{J}{ }_{i} X^{K}{ }_{, j}=-3 C_{I J K} t^{I} t^{J}{ }_{, i} t^{K}{ }_{, j} .
$$

We can express $G_{I J}$ in terms of $t^{I}$ through the equations

$$
G_{I J}=-\frac{6^{1 / 3}}{2}\left(C_{I J}-\frac{3}{2} t_{I} t_{J}\right)
$$

and the inverse metric acting on vectors is

$$
G^{I J}=-\frac{2}{6^{1 / 3}}\left(C^{I J}-3 t^{I} t^{J}\right), \quad G^{I K} G_{K J}=\delta_{J}^{I} .
$$

We will need, in what follows, the relation between the derivative of the special coordinate $\left(t^{I}\right)_{, i}$ and that of the dual coordinate $t_{I, i}$. Using the fact that $C_{I J K}$ is a symmetric numerical tensor we have

$$
t_{I, i}=2 C_{I J K}\left(t^{J}\right)_{, i} t^{K}=2 C_{I J}\left(t^{J}\right)_{, i}, \quad\left(t^{J}\right)_{, i}=\frac{1}{2} C^{I J} t_{I, i} .
$$

Differentiating Eqs. (9) we get

$$
C_{I J K} t^{I} t^{J}\left(t^{K}\right)_{, i}=0, \quad t_{I}\left(t^{I}\right)_{, i}=t_{I, i} t^{I}=0 .
$$

The supersymmetry transformation laws are

$$
\begin{gathered}
\delta e_{\mu}{ }^{m}=\frac{1}{2} \bar{\epsilon} \Gamma^{m} \psi_{\mu}, \\
\delta \psi_{\mu}=D_{\mu}(\hat{\omega}) \epsilon+\frac{i}{8 \cdot 6^{1 / 3}} t_{I}\left(\Gamma_{\mu}{ }^{\nu \rho}-4 \delta_{\mu}{ }^{\nu} \Gamma^{\rho}\right) \hat{F}_{\mu \nu}{ }^{I} \epsilon \\
+\frac{1}{12} g_{i j}\left(\frac{1}{4} \Gamma_{\mu \nu \rho} \epsilon \bar{\lambda}^{i} \Gamma^{\nu \rho} \lambda^{j}-\Gamma_{\mu \nu} \epsilon \bar{\lambda}^{i} \Gamma^{\nu} \lambda^{j}\right. \\
\left.-\Gamma^{\nu} \epsilon \bar{\lambda}^{i} \Gamma_{\mu \nu} \lambda^{j}+2 \epsilon \bar{\lambda}^{i} \Gamma_{\mu} \lambda^{j}\right), \\
\delta \lambda_{i}=\delta \phi^{k} \Gamma_{k i}{ }^{l} \lambda_{l}+\frac{1}{4}\left(\frac{3}{4}\right)^{2 / 3} t_{I, i} \Gamma^{\mu \nu} \epsilon F_{\mu \nu}^{I}-\frac{i}{2} g_{i j} \Gamma^{\mu} \partial_{\mu} \phi^{j} \epsilon \\
+\frac{3 i}{16} t^{I}{ }_{i} t^{J}{ }_{, j} t^{K}{ }_{, k}\left(-3 \epsilon \bar{\lambda}^{j} \lambda^{k}+\Gamma^{\mu} \epsilon \bar{\lambda}^{j} \Gamma^{\mu} \lambda^{k}\right. \\
\left.+\frac{1}{2} \Gamma_{\mu \nu} \epsilon \bar{\lambda}^{j} \Gamma^{\mu \nu}{ }^{\mu \nu} \lambda^{k}\right), \\
{ }^{1 / 3}\left(\frac{1}{2} t^{I}{ }_{, i} \epsilon \Gamma_{\mu} \lambda^{i}+i \bar{\psi} \epsilon t^{I}\right), \\
\delta \phi^{i}=\frac{i}{2} \overline{\epsilon \lambda^{i} .}
\end{gathered}
$$

\section{NEAR HORIZON GEOMETRY AND SUPERSYMMETRY}

Double-extreme black holes (which have 1/2 of unbroken supersymmetry and have constant moduli [14]) in five dimensions have the geometry of the extreme Tangherlini solution [15]. It is a 5D analogue of the extreme ReissnerNordstrom metric:

$$
d s^{2}=-\left[1-\left(\frac{r_{0}}{r}\right)^{2}\right]^{2} d t^{2}+\left[1-\left(\frac{r_{0}}{r}\right)^{2}\right]^{-2} d r^{2}+r^{2} d \Omega_{3}^{2}
$$

and

$$
2 \sqrt{-g} G_{I J} F_{t r}^{J}=q_{I}, \quad \phi^{i}=\text { const. }
$$

The horizon is at $r=r_{0}$ where the parameter $r_{0}$ defining the horizon as well as the constant values of moduli depend on charges of the vector fields and on the topological intersection form $C_{I J K}$. Our study of the near horizon geometry will allow us to determine this dependence.

Note that the area of the horizon of the black hole is given by the volume of the three-dimensional sphere

$$
A=2 \pi^{2} r_{0}^{3} .
$$

The area formula of 5D black holes was found in [9] from the observation that the unbroken supersymmetry near the black hole horizon requires that the central charge $Z \sim t^{I} q_{I}$ has to be extremized in the moduli space, i.e., near the horizon $\partial_{i} Z=0$. This leads to the area formula in the form $A \sim\left(\left.q_{I} q_{J} C^{I J}\right|_{\partial_{i} Z=0}\right)^{3 / 4}$, where $C^{I J}$ is the inverse of $C_{I J}=C_{I J K} t^{K}$. In the derivation of this area formula it was assumed that the unbroken supersymmetry of the black hole solution is enhanced near the horizon. This will be proved now. It will be also explained why the enhancement of supersymmetry near the horizon requires the extremization of the central charge for describing the area formula. By exactly solving the Killing equations for the near horizon geometry we will be able to justify the area formula suggested in [9] and find the explicit area formula including the numerical factor in front of it.

Near the horizon at $r \rightarrow r_{0}$ and one can exhibit the $\mathrm{AdS}_{2} \times S^{3}$ geometry using $\hat{r}=\left(r-r_{0}\right) \rightarrow 0$

$$
d s^{2}=-\left(\frac{2 \hat{r}}{r_{0}}\right)^{2} d t^{2}+\left(\frac{2 \hat{r}}{r_{0}}\right)^{-2} d \hat{r}^{2}+r_{0}^{2} d \Omega_{3}^{2} .
$$

Since we deal with the product space we may use $a=0,1$ for the coordinates of the $\mathrm{AdS}_{2}$ space and $\alpha=2,3,4$ for the coordinates of the three-sphere (in tangent space). The vector field ansatz near the horizon becomes

$$
2\left(r_{0}\right)^{3} G_{I J} F_{a b}^{J}=\epsilon_{a b} q_{I} .
$$

Let us use this ansatz in the fermionic part of supersymmetry transformations with all vanishing fermions and constant moduli. We start with gaugino and keep only relevant terms

$$
\delta \lambda_{i}=\frac{1}{4}\left(\frac{3}{4}\right)^{2 / 3} t_{I, i} \Gamma^{\mu \nu} \epsilon F_{\mu \nu}^{I}=0 .
$$


We study the possibility that the zero mode of this equation is given by the full size spinor $\epsilon$ without linear constraints on it, i.e., that the unbroken supersymmetry is indeed enhanced near the horizon. This is possible, provided that

$$
t_{I, i} G^{I J} q_{J}=0 .
$$

The enhancement of unbroken supersymmetry near the horizon which can be deduced from the gaugino part of supersymmetry, can be represented as the condition of the minimization of the central charge, as found in [9]. Let us derive this here in a more detailed way. The gravitino transformation

$$
\delta \psi_{\mu}=D_{\mu}(\omega) \epsilon+\frac{i}{8 \cdot 6^{1 / 3}} t_{I}\left(\Gamma_{\mu}^{\nu \rho}-4 \delta_{\mu}{ }^{\nu} \Gamma^{\rho}\right) F_{\mu \nu}{ }^{I} \epsilon=0
$$

shows that the graviphoton field strength is given by the linear combination of vector fields and moduli $t_{I} F_{\mu \nu}$, and therefore the central charge is proportional to $t^{I} q_{I}$. From Eq. (25) we get

$$
t_{I, i}\left(C^{I J}-3 t^{I} t^{J}\right) q_{J}=0 .
$$

Using Eqs. (17) and (18) we can conclude that

$$
t_{I, i}\left(C^{I J}-3 t^{I} t^{J}\right) q_{J}=2\left(t^{J}\right)_{, i} q_{J}=0 \Rightarrow \partial_{i} Z=0 .
$$

Thus we have derived the condition of minimization of the central charge $Z=t^{I} q_{I}$ in the moduli space from the requirement that the gaugino supersymmetry transformation for constant moduli has the full size spinor $\epsilon$ as a zero mode, i.e., from the condition of enhancement of supersymmetry near the black hole horizon. The central charge has to be independent of $\phi^{i}$. Let us consider some useful identities of the real special geometry which are valid only near horizon. Note that

$$
g^{i j} \partial_{i} Z \partial_{j} Z=g^{i j} t_{, i}^{I} t_{, j}^{J} q_{I} q_{J} \equiv \Pi^{I J} q_{I} q_{J}=0 .
$$

Using Eq. (18) we find that

$$
\Pi^{I J} t_{I}=0
$$

and we may look for the combination which is orthogonal to $t_{I}$ in the form $\Pi^{I J}=l\left(C^{I J}-t^{I} t^{J}\right)$. To get the coefficient $l$ we use $g_{i j}=6^{2 / 3} t_{, i}{ }_{i}{ }^{J}{ }_{, j} G_{I J}$ and contract it with $g^{i j}$ :

$$
g_{i j} g^{i j}=h_{(1,1)}-1=6^{2 / 3} \Pi^{I J} G_{I J},
$$

which leads to $l=-\frac{1}{3}$. Thus we conclude that near the horizon where the central charge is moduli independent we have an identity

$$
\left[\left(C^{I J}-t^{I} t^{J}\right) q_{I} q_{J}\right]_{\partial_{i} Z=0}=0 .
$$

What remains to be done to make the extremization of the central charge consistent is to check what happens with the gravitino: does the gravitino transformation rule admit the Killing spinor of the full supersymmetry in our background? And what are the conditions of that? Using the ansatz for the vector fields near the horizon (23) and taking into account that we have a product space we get the following form of the gravitino transformations (26):

$$
\begin{gathered}
\delta \psi_{a}=\left(\hat{\nabla}_{a}\right) \eta \equiv\left(\nabla_{a}+c \tilde{\gamma}_{a}\right) \eta=0, \quad a=0,1, \\
\delta \psi_{\alpha}=\left(\hat{\nabla}_{\alpha}\right) \varsigma \equiv\left(\nabla_{\alpha}-\frac{c}{2} \gamma_{\alpha}\right) \varsigma=0, \quad \alpha=2,3,4,
\end{gathered}
$$

where

$$
\tilde{\gamma}_{a}=i \epsilon_{a b} \gamma^{b}, \quad \Gamma^{a}=\gamma^{a} \otimes I, \quad \Gamma_{\alpha}=i \gamma_{0} \gamma_{1} \otimes \gamma_{\alpha},
$$

and

$$
c=-\frac{1}{6^{2 / 3}} \frac{t^{I} q_{I}}{r_{0}^{3}},
$$

and the $\gamma$ matrices satisfy

$$
\begin{gathered}
\left\{\tilde{\gamma}_{a}, \tilde{\gamma}_{b}\right\}=2 \eta_{a b}, \quad \eta_{a b}=(-,+), \quad \tilde{\gamma}_{[a b]}=-\gamma_{[a b]}, \\
\left\{\gamma_{\alpha}, \gamma_{\beta}\right\}=2 \delta_{a b}, \quad \alpha=2,3,4 .
\end{gathered}
$$

This is a special example of the general case presented in the Introduction. We have identified the supersymmetric Killing spinor with the geometric Killing spinor. The integrability conditions for the geometric Killing spinors defined above are

$$
\begin{aligned}
{\left[\hat{\nabla}_{a}, \hat{\nabla}_{b}\right] \eta(x)=0 } & \Rightarrow R_{a b}{ }^{c d}=\frac{4}{6^{4 / 3}} \frac{\left(t^{I} q_{I}\right)^{2}}{r_{0}^{6}}\left(\delta_{a}^{c} \delta_{b}{ }^{d}-\delta_{a}{ }^{d} \delta_{b}{ }^{c}\right), \\
{\left[\hat{\nabla}_{\alpha}, \hat{\nabla}_{\beta}\right] \varsigma(y) } & =0 \Rightarrow R_{\alpha \beta}{ }^{\gamma \delta} \\
& =-\frac{1}{6^{4 / 3}} \frac{\left(t^{I} q_{I}\right)^{2}}{r_{0}^{6}}\left(\delta_{\alpha}^{\gamma} \delta_{\beta}{ }^{\delta}-\delta_{\alpha}{ }^{\delta} \delta_{\beta}{ }^{\gamma}\right)
\end{aligned}
$$

This can be contracted to give us the Ricci tensors on $\mathrm{AdS}_{2}$ and on $S^{3}$ :

$$
R_{a b}=\frac{4}{6^{4 / 3}} \frac{\left(t^{I} q_{I}\right)^{2}}{r_{0}^{6}} \eta_{a b}, \quad R_{\alpha \beta}=-\frac{2}{6^{4 / 3}} \frac{\left(t^{I} q_{I}\right)^{2}}{r_{0}^{6}} \delta_{\alpha \beta},
$$

with the result that the radii of $\mathrm{AdS}_{2}$ and $S^{3}$ are related:

$$
R_{(2)}=-\frac{4}{3} R^{(3)} .
$$

This relation restricts the properties of geometric Killing spinors. They would exist without any relation between these two product geometries. However, supersymmetric Killing 
spinors require relation between geometries. The curvature can be calculated also directly from the metric (22):

$$
R_{a b}=\frac{4}{r_{0}^{2}} \eta_{a b}, \quad R_{\alpha \beta}=-\frac{2}{r_{0}^{2}} \delta_{\alpha \beta} .
$$

Comparing Eqs. (40) with Eqs. (42), we can express $r_{0}$ via the values of the moduli near the horizon and electric charges as

$$
r_{0}^{4}=\frac{1}{6^{4 / 3}}\left[\left(t^{I} q_{I}\right)_{\partial i Z=0}\right]^{2} .
$$

This gives us the area of the horizon

$$
A=2 \pi^{2} r_{0}^{3}=\frac{\pi^{2}}{3}\left\{\left(t^{I} t^{J} q_{I} q_{J}\right)_{\partial i Z=0}\right\}^{3 / 4}
$$

Near the horizon at $\partial i Z=0$ we can rewrite it as

$$
A=\frac{\pi^{2}}{3}\left\{\left(C^{I J} q_{I} q_{J}\right)_{\partial i Z=0}\right\}^{3 / 4}
$$

using identity (32).

Thus we have combined the supersymmetry analysis with the analysis of the geometry. In this way we have confirmed the structure of the area formula of five-dimensional black holes in $N=2$ theories obtained in [9] and found the exact numerical coefficient in front of it for supersymmetric five dimensional black holes with finite area of the horizon in the $N=2$ theory.

\section{TRUNCATION OF $N=4$ SUPERGRAVITY TO $N=2$}

In some cases it is useful to consider those $N=2$ theories which can be obtained by truncation from $N=4$ theories. We will first study the truncation of pure $N=4$ supergravity and later generalize the result to the case of $N=4$ supergravity interacting with arbitrary number of vector multiplets as it was done before in $4 \mathrm{D}$ theories in [10].

We focus on a a special example of the double extreme five-dimensional black hole known as the Strominger-Vafa black hole [6]. The Lagrangian which allows a supersymmetric embedding of this black hole is obtained most easily by the truncation of $N=4$ supergravity in $d=5$ constructed by Awada and Townsend [17]. The bosonic part of the action is

$$
\begin{aligned}
e^{-1} \mathcal{L}= & -\frac{1}{2} R-\frac{1}{4} e^{(2 / 3) \phi} F_{\mu \nu}{ }^{i j} F_{i j}{ }^{\mu \nu}-\frac{1}{4} e^{-(4 / 3) \phi} G_{\mu \nu} G^{\mu \nu} \\
& -\frac{1}{6}\left(\partial_{\mu} \phi\right)^{2}+\frac{e^{-1}}{4 \sqrt{2}} \epsilon^{\mu \nu \rho \sigma \lambda} F_{\mu \nu}{ }^{i j} F_{\rho \sigma i j} B_{\lambda}
\end{aligned}
$$

where $i, j=1, \ldots, 4$ and $G_{\mu \nu}$ is the field strength of $B_{\mu}$ and $F_{\mu \nu}{ }^{i j}$ is the field strength of $A_{\mu}{ }^{i j}$. The supersymmetry transformation laws are

$$
\begin{gathered}
\delta e_{\mu}{ }^{m}=\frac{1}{2} \bar{\epsilon}^{i} \Gamma^{m} \psi_{\mu}, \\
\delta \psi_{\mu i}=D_{\mu}(\hat{\omega}) \epsilon_{i}+\frac{i}{6}\left(e^{(\phi / 3)} F_{\rho \sigma i j}-\frac{1}{2 \sqrt{2}} e^{-(2 / 3) \phi} G_{\rho \sigma} \Omega_{i j}\right) \\
\times\left(\Gamma_{\mu}{ }^{\rho \sigma}-4 \delta_{\mu}{ }^{\rho} \Gamma^{\sigma}\right) \epsilon^{j}+\cdots, \\
\delta A_{\mu}^{i j}=-\frac{1}{\sqrt{3}} e^{-\phi / 3}\left(\bar{\epsilon}^{i i} \Gamma_{\mu} \chi^{j]}+\frac{1}{4} \Omega^{i j} \bar{\epsilon}^{k} \Gamma_{\mu} \chi_{k}\right) \\
-i e^{-(\phi / 3)}\left(\vec{\epsilon}^{i j} \psi_{\mu}^{j]}+\frac{1}{4} \Omega^{i j} \bar{\epsilon}^{k} \psi_{\mu k}\right), \\
\delta B_{\mu}=\frac{1}{\sqrt{6}} e^{2 \phi / 3}\left(\bar{\epsilon}^{i} \Gamma_{\mu} \chi_{i}-\frac{\sqrt{3}}{2} \bar{\epsilon}^{i} \psi_{\mu i}\right), \\
\delta \chi_{i}=-\frac{i}{2 \sqrt{3}} \Gamma^{\mu} \partial_{\mu} \phi \epsilon_{i}+\frac{1}{2 \sqrt{3}} \\
\times\left(e^{\phi / 3} F_{\rho \sigma i j}+\frac{1}{\sqrt{2}} e^{-2 \phi / 3} G_{\rho \sigma} \Omega_{i j}\right) \Gamma^{\rho \sigma} \epsilon^{j}+\ldots \\
\delta \phi=\frac{\sqrt{3}}{2} \bar{\epsilon}^{i} \chi_{i} .
\end{gathered}
$$

Here $\Omega_{i j}$ is a symplectic matrix. The truncation of $N=4$ supergravity to $N=2$ supergravity interacting with one vector multiplet is carried out by keeping $\psi_{\mu i}, \chi_{i}$ with $(i=1,2)$ only and $A_{\mu}{ }^{12}=-A_{\mu}{ }^{34} \equiv \frac{1}{2} A_{\mu}, B_{\mu}, g_{\mu \nu}$ and $\phi$. By inspecting the supersymmetry transformations of the truncated fields it is not difficult to see that this is a consistent truncation. The bosonic action becomes ${ }^{1}$

$$
\begin{aligned}
e^{-1} \mathcal{L}= & -\frac{1}{2} R-\frac{1}{4} e^{(2 / 3) \phi} F_{\mu \nu} F^{\mu \nu}-\frac{1}{4} e^{-(4 / 3) \phi} G_{\mu \nu} G^{\mu \nu} \\
& -\frac{1}{6}\left(\partial_{\mu} \phi\right)^{2}+\frac{e^{-1}}{4 \sqrt{2}} \epsilon^{\mu \nu \rho \sigma \lambda} F_{\mu \nu} F_{\rho \sigma} B_{\lambda}
\end{aligned}
$$

The supersymmetry transformations of the fermionic fields with vanishing fermion are

$$
\begin{aligned}
\delta \psi_{\mu}= & D_{\mu}(\omega) \epsilon-\frac{i}{12}\left(\Gamma_{\mu}{ }^{\rho \sigma}-4 \delta_{\mu}{ }^{\rho} \Gamma^{\sigma}\right) \\
& \times\left(e^{(\phi / 3)} F_{\rho \sigma}-\frac{1}{\sqrt{2}} e^{-(2 / 3) \phi} G_{\rho \sigma}\right) \epsilon, \\
\delta \chi= & -\frac{i}{2 \sqrt{3}} \Gamma^{\mu} \partial_{\mu} \phi \epsilon-\frac{1}{4 \sqrt{3}} \Gamma^{\rho \sigma}\left(e^{\phi / 3} F_{\rho \sigma}\right. \\
& \left.+\sqrt{2} e^{-2 \phi / 3} G_{\rho \sigma}\right) \epsilon,
\end{aligned}
$$

\footnotetext{
${ }^{1}$ This action is equivalent upon rescalings to the action of $N=2$ supergravity interacting with one vector multiplet as presented in the Appendix of [16].
} 
and we have omitted the symplectic index on the spinors (to avoid confusion with the index on $\phi^{i}$ in the previous section).

Our action can be compared with the action used in [6] if we rewrite it as follows:

$$
\begin{aligned}
e^{-1} \mathcal{L}= & \frac{1}{2}\left(-R-\frac{1}{4} e^{(2 / 3) \phi} F_{\mu \nu}^{\prime} F^{\prime} \mu \nu-\frac{1}{4} e^{-(4 / 3) \phi} G_{\mu \nu}^{\prime} G^{\prime \mu \nu}\right. \\
& \left.-\frac{1}{3}\left(\partial_{\mu} \phi\right)^{2}+\frac{e^{-1}}{8} \epsilon^{\mu \nu \rho \sigma \lambda} F_{\mu \nu}^{\prime} F_{\rho \sigma}^{\prime} B_{\lambda}^{\prime}\right)
\end{aligned}
$$

where

$$
F^{\prime}=\sqrt{2} F, \quad G^{\prime}=\sqrt{2} G, \quad B^{\prime}=\sqrt{2} B .
$$

We observe a discrepancy in the kinetic term for the scalar field which is, however, harmless since the solution has a constant moduli field. Thus the extreme black hole of Strominger-Vafa can be embedded into the $N=2$ supergravity interacting with one constant vector multiplet.

To make contact with the formalism of previous section dealing with the general case of the very special geometry, we make the following identifications: $i=1, I=0,1$ and

$$
\begin{gathered}
i=1, \quad I=0,1, \quad A_{\mu}{ }^{1} \equiv A_{\mu}, \quad A_{\mu}{ }^{0} \equiv B_{\mu} \\
G_{11}=e^{2 \phi / 3}, \quad G_{00}=e^{-(4 \phi / 3)}, \quad C_{011}=2 \sqrt{2}, \quad g_{11}=1, \\
\phi^{1}=\frac{1}{\sqrt{3}} \phi, \quad q_{1}=-\frac{8 \sqrt{2} Q_{F}}{\pi}, \quad q_{0}=2 \sqrt{2} Q_{H} .
\end{gathered}
$$

Comparing the supersymmetry transformations we must identify

$$
\frac{\partial t_{1}}{\partial \phi^{1}}=-\frac{1}{\sqrt{3}}\left(\frac{4}{3}\right)^{2 / 3} e^{\phi / 3}, \quad \frac{\partial t_{0}}{\partial \phi^{1}}=-\sqrt{\frac{2}{3}}\left(\frac{4}{3}\right)^{2 / 3} e^{-2 \phi / 3} .
$$

To satisfy the relations of special geometry and in particular to have $\left(t^{I}\right),{ }_{i} t_{I}=0$ we get

$$
\begin{gathered}
t_{1}=-\left(\frac{4}{3}\right)^{2 / 3} e^{\phi / 3}, \quad t^{1}=\frac{2}{3}\left(\frac{3}{4}\right)^{2 / 3} e^{-\phi / 3}, \\
t_{0}=\frac{1}{\sqrt{2}}\left(\frac{4}{3}\right)^{2 / 3} e^{-2 \phi / 3}, \quad t^{0}=\sqrt{\frac{2}{3}}\left(\frac{3}{4}\right)^{2 / 3} e^{2 \phi / 3} .
\end{gathered}
$$

The enhancement of supersymmetry near the horizon is provided by $\left(\partial t_{I} / \partial \phi\right) G^{I J} q_{J}=0$ and we get the fixed value of the moduli near the horizon:

$$
\begin{gathered}
e^{\phi}=-\frac{1}{\sqrt{2}} \frac{q_{1}}{q_{0}}, \quad t^{1} q_{1}=\frac{1}{2^{1 / 6} 3^{1 / 3}}\left[q_{0}\left(q_{1}\right)^{2}\right]^{1 / 3}, \\
t^{0} q_{0}=\frac{1}{2} t^{1} q_{1} .
\end{gathered}
$$

Now we can express the combination $t^{I} q_{I}$ near the horizon required for the entropy as

$$
t^{I} q_{I}=\frac{3^{2 / 3}}{2^{7 / 6}}\left[q_{0}\left(q_{1}\right)^{2}\right]^{1 / 3}
$$

and

$$
r_{0}^{6}=\frac{1}{6^{2}}\left\{\left(t^{I} q_{I}\right)_{\mathrm{hor}}\right\}^{3} \Rightarrow \frac{1}{2^{11 / 2}}\left(-q_{0}\right)^{2} q_{1}=\frac{8\left(Q_{H} Q_{F}\right)^{2}}{\pi^{2}},
$$

and

$$
A=2 \pi^{2}\left(r_{0}\right)^{3}=\frac{\pi^{2}}{2}\left(\frac{q_{0}}{\sqrt{2}}\left[\frac{q_{1}}{\sqrt{2}}\right]^{2}\right)^{1 / 2} .
$$

Thus we have reproduced the Strominger-Vafa area formula as an example of our general area formula

$$
A=2 \pi^{2}\left(r_{0}\right)^{3}=\frac{\pi^{2}}{3}\left\{\left(C^{I J} q_{I} q_{J}\right)_{\partial i Z=0}\right\}^{3 / 4} \Rightarrow 8 \pi \sqrt{\frac{Q_{H}\left(Q_{F}\right)^{2}}{2}},
$$

and therefore this black hole solution fits into our consideration of the very special geometry.

For the general case of $n, N=4$ vector multiplets with duality group $\mathrm{O}(1,1) \times \mathrm{O}(5, n) /[\mathrm{O}(5) x] \mathrm{O}(n)$ the formula for the largest eigenvalue of the central charge, extremized in the moduli space was presented in [10]. This translates into the $N=4$ duality invariant area formula

$$
A=8 \pi \sqrt{\frac{Q_{H}\left(Q_{F}\right)^{2}}{2}},
$$

where $Q_{H}$ is the singlet charge and $\left(Q_{F}\right)^{2}$ is the $O(5, n)$ Lorenzian norm of the other $5+n$ charges $Q_{F}$.

Upon truncation to $N=2$ theory this gives a very special geometry with $C_{I J K}$ intersection of the form $(I, J=0,1, \ldots, n+1)$ and $(i=1, \ldots, n+1)$

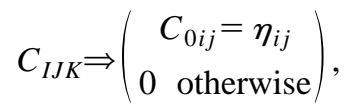

where $\eta_{i j}$ is the Lorenzian metric of $\mathrm{O}(1, n)$ and the very special geometry is $\mathrm{O}(1,1) \times \mathrm{O}(1, n) / \mathrm{O}(n)$. Upon truncation the area has the same form as in Eq. (60),

$$
A=8 \pi \sqrt{\frac{Q_{H}\left(Q_{F}\right)^{2}}{2}}=8 \pi \sqrt{\frac{Q_{H}\left(Q_{i} \eta_{i j} Q_{j}\right)}{2}},
$$

where again $Q_{H}$ is the singlet charge and $\left(Q_{F}\right)^{2}$ is the $\mathrm{O}(1, n)$ Lorenzian norm of the other $1+n$ charges $Q_{i}$.

\section{DISCUSSION}

Thus we have given a complete description of 5D static black holes near the horizon which can be embedded into $N=2$ and $N=4$ supergravity with arbitrary number of vector multiplets. They all show enhancement of supersymmetry near the black hole horizon. In the $N=2$ as well as in the $N=4$ case, the unbroken supersymmetry $(1 / 2$ in $N=2$ and $1 / 4$ in $N=4)$ is doubled. As the result in all cases the dimension of the Killing spinor of unbroken supersymmetry is the 
dimension of the Dirac spinor admitted by the anti-de Sitter space and the sphere.

The significance of the enhancement of supersymmetry near the horizon is related to the fact that in all cases when we have found finite horizon area of supersymmetric solutions there was also the enhancement of supersymmetry present. This concerns all four- and five-dimensional static black holes where we deal with the near-horizon geometries $\mathrm{AdS}_{2} \times S^{2}$ and $\mathrm{AdS}_{2} \times S^{3}$, respectively. The area in both cases is given by the volume of the $S^{2}$ and $S^{3}$ sphere.

In more general situations when the near horizon geometry is given by $\operatorname{AdS}_{p+2} \times S^{d-p-2}$, the area of the horizon is given by the volume of the $S^{d-p-2}$ sphere times the volume of the torus of dimension $p$, which for the supersymmetric solutions shrinks to zero near the horizon. As the result, in the class of solutions with $\operatorname{AdS}_{p+2} \times S^{d-p-2}$ near-horizon geometries one cannot expect finite area of the horizon per unit volume except for black holes. Examples of such configurations with enhancement of supersymmetry and still vanishing area of the horizon include: $d=10, p=3$; $d=11, p=2, p=5$. This geometric observation matches the recent analysis [19] of the cases of the vanishing entropy in which a duality invariant expression in terms of integral charges does not exist. In all these situations the extremum of the central charge does not occur at finite rational values of the moduli.

Few more configurations with near-horizon geometry $\operatorname{AdS}_{p+2} \times S^{d-p-2}$ are known [18]. They include $\mathrm{AdS}_{3} \times S^{2}$ describing a 5D magnetic string and $\mathrm{AdS}_{3} \times S^{3}$ describing a $6 \mathrm{D}$ self-dual string. It has not been established yet whether they have enhancement of supersymmetry near the horizon. A calculation of the type which we have performed here for $5 \mathrm{D}$ black holes is required to identify the supersymmetric
Killing spinors with the geometric ones for the $5 \mathrm{D}$ and $6 \mathrm{D}$ strings.

From the perspective of this study it would be interesting to have a more careful look into configurations with $\operatorname{AdS}_{2} \times S^{d-2}$ geometries near the horizon. These would have finite nonvanishing area related to the volume of the $S^{d-2}$ sphere. Such configurations of the Reissner-NordstromTangherlini type are known to solve equations of motion of Einstein-Maxwell theory in any dimension. ${ }^{2}$ However in $d>5$ they do not seem to have a supersymmetric embedding, at least such embeddings have not been found so far.

In conclusion, we have studied the mechanism of enhancement of unbroken supersymmetry near the 5D black hole horizon and we have found the entropy-area formula for solutions of $N=2$ supergravity interacting with arbitrary number of vector multiplets.

\section{ACKNOWLEDGMENTS}

We are grateful to Gary Horowitz, Andrew Strominger, Amanda Peet, Tomás Ortín, Arvind Rajaraman, Paul Townsend, and Wing Kai Wong for stimulating discussions. The work of S.F. was supported in part by U.S. DOE Grant No. DE-FGO3-91ER40662 and by European Commission TMR program No. ERBFMRX-CT96-0045. G.W.G. would like to thank N. Straumann for hospitality and the Swiss National Science Foundation for financial support at ITP, Zurich where part of this work was carried out. R.K. was supported by the NSF Grant No. PHY-8612280 and by the Institute of Theoretical Physics (ETH) in Zurich and CERN where part of this work was done.

\footnotetext{
${ }^{2}$ We are grateful to G. Horowitz for this observation.
}

[1] G. W. Gibbons, Nucl. Phys. B207, 337 (1982).

[2] R. Kallosh and A. Peet, Phys. Rev. D 46, 5223 (1992).

[3] G. W. Gibbons and P. K. Townsend, Phys. Rev. Lett. 71, 3754 (1993).

[4] R. Kallosh, Phys. Lett. B 282, 80 (1992).

[5] G. W. Gibbons, D. Kastor, L. A. J. London, P. K. Townsend, and J. Traschen, Nucl. Phys. B416, 850 (1994).

[6] A. Strominger and C. Vafa, Phys. Lett. B 379, 99 (1996).

[7] J. Beckenridge, R. Myers, A. Peet, and C. Vafa, "D-Branes and Spinning Black Holes," Harvard University Report No. HUTP-96-A005, hep-th/9602065, 1996 (unpublished).

[8] A. A. Tseytlin, Mod. Phys. Lett. A 11, 689 (1996); M. Cvetic and D. Youm, Nucl. Phys. B476, 118 (1996).

[9] S. Ferrara and R. Kallosh, Phys. Rev. D 54, 1514 (1996).

[10] S. Ferrara and R. Kallosh, Phys. Rev. D 54, 1525 (1996).

[11] M. Günaydin, G. Sierra, and P. K. Townsend, Nucl. Phys. B242, 244 (1984); B253, 573 (1985).
[12] B. de Wit and A. van Proyen, Phys. Lett. B 293, 94 (1992).

[13] A. C. Cadavid, A. Ceresole, R. D'Auria, and S. Ferrara, Phys. Lett. B 357, 76 (1995); G. Papadopoulos and P. K. Townsend, ibid. 357, 300 (1995); I. Antoniadis, S. Ferrara, and T. R. Taylor, Nucl. Phys. B460, 489 (1996).

[14] R. Kallosh, M. Shmakova, and W. K. Wong, Phys. Rev. D 54, 6289 (1996).

[15] F. R. Tangherlini, Nuovo Cimento 27, 636 (1963); R. C. Myers and M. J. Perry, Ann. Phys. (N.Y.) 172, 304 (1986).

[16] G. W. Gibbons and P. K. Townsend, Phys. Lett. B 356, 472 (1995).

[17] M. Awada and P. K. Townsend, Nucl. Phys. B255, 617 (1985).

[18] G. W. Gibbons, G. Horowitz, and P. K. Townsend, Class. Quantum Grav. 12, 297 (1995).

[19] L. Andrianopoli, R. D'Auria, and S. Ferrara, “General Extension of Extended Supergravity in Diverse Dimensions,', Report No. hep-th/9608015 (unpublished). 